\title{
COVID-19 Causing Persistent Cacosmia In A pregnant Patient: First Case Report.
}

\author{
Lina Okar ${ }^{1}$, Eman Abdelkarim ${ }^{1}$, Rasha Moussa $^{1}$, and Muna Aseel ${ }^{1}$ \\ ${ }^{1}$ Hamad Medical Corporation
}

November 26, 2020

\begin{abstract}
Olfactory and gustatory dysfunctions were prescribed in COVID-19 infection. Monitoring and early detection for COVID-19 infection in pregnancy is essential. Keeping rare presentations and the impact they have on the pregnancy in mind is crucial.This is the first case of pregnant woman who had cacosmia as persistent symptoms after infection
\end{abstract}

\section{Introduction:}

Due to the widely spread of the recent virus SARS-Cov2, a lot of studies described the clinical manifestations dividing them into typical and atypical or common and uncommon. While upper respiratory tract symptoms were under the common ones the gastrointestinal symptoms were not[1]. Also, olfactory, and gustatory dysfunctions were prescribed in the literature. The clinical course of COVID-19 infections varies among patients, people who were considered vulnerable had worse clinical progress[2]. Pregnant women were among the vulnerable population mainly because coronavirus is new, and its outcomes are unpredictable. However, pregnant women had the same clinical presentations as general population[1]. Here we present the first case report of a 32-year-old pregnant women infected with COVID-19 and had a unique persistent symptom which is cacosmia. We are discussing the impact of such underestimated symptoms on the pregnancy.

\section{Case presentation:}

32 year-Old Egyptian female gravida 3, Para 2 (G3,P2) week 23 presented to the Clinic in the primary health care center on June,2020 complaining from three days loss of smell and taste, fatigue, no fever, no respiratory or gastrointestinal symptoms. She had history of sick contact with her husband. The patient past medical history was remarkable for gestational diabetes in her second pregnancy, she is not smoker. Covid19 PCR from Nasopharyngeal swab was positive with low antibodies level according to our local guideline which indicate quarantine. In August 2020, the patient was contacted via phone consultation, she was 28 weeks on her pregnancy, her main complaint was cacosmia that causes her nausea and vomiting sometimes, she described her complain as unpleasant sensation started 6 weeks ago, her other symptoms improved after few days from COVID-19 diagnosis yet the smell disturbances persist, it was bilateral, continuous, without discharge, sometimes as foul odor, the smell was not noticed by her surroundings, increases when smelling potent odors like perfumes, soups or cleaners. She had no previous history of any type of rhinitis, asthma, or any atopic related diagnosis. Her primary laboratory results were normal except low vitamin B12 (table.1). She was seen later by ENT, examination showed pale nasal mucosa, Mometasone nasal spray was prescribed. However, further imagining was postponed due to the pregnancy state. In October 2020 she was seen again after normal delivery and sinus Xray did not reveal any signs of chronic sinusitis, she was still complaining from cacosmia which unfortunately did not improve with the symptomatic treatment and intra-nasal corticosteroids. Currently, we are still following up the patient and she is still complaining from cacosmia. 


\section{Discussion:}

Severe acute respiratory syndrome coronavirus-2 (SARS-COV-2) is very contagious, that why it spread rapidly around the world since the end of 2019 causing the most recent respiratory pandemic in our century. History that might reveal exposure risk for diagnosed cases as well clinical manifestations plays an important role in the suspicion and thus diagnosis cases and controlling infection spread. Diagnosis confirmation is mainly via reverse transcriptase chain reaction (RT-PCR) though it has $30 \%$ false negative rate. Clinical manifestations might vary widely. However, the most common ones are fever, fatigue, headache, and myalgia. Gastrointestinal symptoms were considered less common[1]. Other atypical presentation like anosmia (Loss of smell), Agnosia (Loss of taste) were reported before[3].

The impact of coronavirus on pregnant women is not clear till today. However, some studies consider pregnancy as vulnerable state and viral infections might increase morbidity and mortality in the mother as well her fetus yet no evidence to our date that pregnancy increases COVID-19 susceptibility and risk of COVID-19 complications[1]. During pregnancy the symptoms were as described among non-pregnant patients. However, our patient has smooth course of infection and normal delivery without complication. Cacosmia was the only persistent symptoms that cause her nausea and few times vomiting during the second and third trimester which obviously affected her psychologically and nutrition.

Recent European study observed the ENT presentation of COVID-19 and found that olfactory and gustatory dysfunction are quite common with olfactory dysfunction occurring in $85.6 \%$ and gustatory dysfunction in $88 \%$ [2]. Another study showed that olfactory loss or anosmia occurs in $39 \%$ following upper respiratory infections with a return to normal rate that varies depending on multiple factors like the patient age, gender and if the baseline disorder is hyposmia or anosmia [4]. Thus, anosmia was suggested to be one of the diagnostic criteria especially in the presence of other symptoms, and most of the patients that were included of this study described that changing on taste and smell happened after other symptoms rather from the beginning. $[5,6]$.However, Cacosmia which happened in our patient was note prescribed in the previously published studies.

Olfactory disturbances are classified into three categories: first, Hyposmia and anosmia which mean decreased sensitivity. Second, odorant stimulation disorders where there is a distortion in the perceived odor (Troposmia), and third, smelling hallucination where the odor is mostly unpleasant (phantosmia), unpleasant phantosmia sometimes called cacosmia. Usually the distortions on the smell is the most upsetting symptoms to the patients and remarkably affects their quality of life among these disturbances. Etiology behind this disorder is not clearly defined but it can be categorized into central and peripheral. Central etiologies or combination of both[7].

To the best of our knowledge, no previously published articles about cacosmia, thought other olfactory disorders like hyposmia and anosmia have been clearly described. Our patient had the gustatory and olfactory symptoms from the beginning. The persistent of hyposmia and cacosmia mainly was very irritant to her, giving her unpleasant feeling during her second and third trimester and causing persistent nausea. By excluding other possible diagnosis, persistent of complain after delivery, not responding to specific treatments, all of these factors made us correlate the patient complain to COVID-19 especially on the absence of similar episodes before the infection. We believe that physician should be aware of the ENT presentation of COVID19 including ageusia and anosmia as well considering cacosmia in COVID-19 patients. Whether pregnancy has a role on the prolongation of this atypical symptoms or not is something that needs further research. Physician as well should take into account the implication of cacosmia on the pregnant women due to the physiological impact and the affect on the nutrition which might indeed affect the pregnancy.

\section{Conclusion:}

With the last worldwide pandemic caused by coronavirus and it is unknown long term outcomes especially on vulnerable categories. Physician should keep close monitoring for pregnant lady infected with this virus and ask about the olfactory and gustatory disturbances. Anosmia, hyposmia were reported previously yet here we report the first case of persistent cacosmia in a pregnant woman that caused a huge distress and caused 
nausea persisted even after delivery. We suggest that cacosmia might be a presenting and persistent symptom among COVID-19 patients and that pregnancy might prolong the course of symptoms. Reassurance and supporting were of an important value in this case due to the psychological impact of the unpleasant smell in our patient quality of life.

\section{Acknowledgment}

We would like to acknowledge Family medicine residency program in Hamad medical corporation for their support and Westbay primary health care center.

Open access funded by Qatar National Library.

\section{Statement of Ethics}

Consent was obtained from the patients.

Case approved by HMC Medical Research center

\section{Declaration of interest}

The authors report no conflicts of interest.

\section{Data availability statement:}

All data related to the article are available upon request.

\section{Authors contribution:}

Lina Okar: Writing the manuscript and literature review.

Eman Abelkarim: Writing the case presentation.

Rasha Moussa and Muna Aseel: Reviewing the manuscript.

\section{References:}

[1] Rajewska A, W M-B, J L-K, M S, S K, A T. COVID-19 and pregnancy - where are we now? A review. J Perinat Med 2020;48:428-34.

[2] Lechien JR, Chiesa-Estomba CM, De Siati DR, Horoi M, Le Bon SD, Rodriguez A, et al. Olfactory and gustatory dysfunctions as a clinical presentation of mild-to-moderate forms of the coronavirus disease (COVID-19): a multicenter European study. Eur Arch Oto-Rhino-Laryngology 2020;277:2251-61. https://doi.org/10.1007/s00405-020-05965-1.

[3] Mak PQ, Chung KS, Wong JSC, Shek CC, Kwan MYW. Anosmia and ageusia: Not an uncommon presentation of COVID-19 infection in children and adolescents. Pediatr Infect Dis J 2020;39:E199-200. https://doi.org/10.1097/INF.0000000000002718.

[4] Temmel AFP, Quint C, Schickinger-Fischer B, Klimek L, Stoller E, Hummel T. Characteristics of olfactory disorders in relation to major causes of olfactory loss. Arch Otolaryngol - Head Neck Surg 2002;128:635-41. https://doi.org/10.1001/archotol.128.6.635.

[5] Giacomelli A, Pezzati L, Conti F, Bernacchia D, Siano M, Oreni L, et al. Self-reported olfactory and taste disorders in patients with severe acute respiratory coronavirus 2 infection: A cross-sectional study. Clin Infect Dis 2020;71:889-90. https://doi.org/10.1093/cid/ciaa330.

[6] Spinato G, Fabbris C, Polesel J, Cazzador D, Borsetto D, Hopkins C, et al. Alterations in Smell or Taste in Mildly Symptomatic Outpatients with SARS-CoV-2 Infection. JAMA - J Am Med Assoc 2020;323:2089-91. https://doi.org/10.1001/jama.2020.6771.

[7] Leopold D. Distortion of olfactory perception: Diagnosis and treatment. Chem Senses 2002;27:611-5. https://doi.org/10.1093/chemse/27.7.611. 
List of figure and table legends:

Table1. 